\title{
Estimation of the True Granulocyte-Macrophage Colony-Stimulating Factor (GM-CSF) Autoantibody Concentration in the Sera of Normal Subjects
}

Keiko Shiiya', Mariko lizuka1, Yuko Ito', Seino Utako', Hashimoto Atsushi', Takahiro Tanaka², Keiichi Akasaka ${ }^{3}$, Nobutaka Kitamura Yoshikazu Inoue ${ }^{4}$, Ryushi Tazawa ${ }^{1}$ and Koh Nakata ${ }^{1,2^{\star}}$

${ }^{1}$ Bioscience Medical Research Center, Niigata University Medical \& Dental Hospital Niigata, Japan

${ }^{2}$ Clinical and Translational Research Center, Niigata University Medical \& Dental Hospital Niigata, Japan

${ }^{3}$ Uonuma General Education Center, Niigata University Medical \& Dental Hospital Niigata, Japan

${ }^{4} \mathrm{NHO}-K i n k i c h u o$ Chest Medical Center, Osaka, Japan

\begin{abstract}
According to our previous studies, Granulocyte-Macrophage Colony-Stimulating Factor (GM-CSF) autoantibodies (GMAbs) are normally present in healthy people. To date, antigen-antibody capture assay has measured the concentrations. However, nonspecific IgG other than GMAbs and immune complex formation with intrinsic GM-CSF prevents us from measuring the accurate concentrations. To solve these problems, we developed a liquid phase high-sensitivity assay using biotinylated GM-CSF (bGM-CSF) and streptavidin coated plate, which could measure the concentration of GMAbs over $0.02 \mathrm{ng} / \mathrm{ml}$. Using this method, the true GMAbs concentration in 30 normal subjects was estimated to be $52.6 \mathrm{ng} / \mathrm{ml}$ for the median value, ranging 10.6 to $799.5 \mathrm{ng} / \mathrm{ml}$. Thus, we propose a novel method that enables us to estimate the true concentration of GMAbs in the sera of healthy subjects.
\end{abstract}

Keywords: Pulmonary alveolar proteinosis; GranulocyteMacrophage Colony-Stimulating Factor (GM-CSF); Autoantibody; Liquid phase reaction

\section{Introduction}

In 1999, we discovered high concentrations of GMAbs in both sera and bronchoalveolar lavage fluids from patients with autoimmune pulmonary alveolar proteinosis (aPAP) that was called "idiopathic pulmonary alveolar proteinosis" at that time $[1,2]$. These autoantibodies showed high avidity, specificity, and neutralizing capacity to GM-CSF and had multiple epitopes on the molecule [3]. As gene-targeting mice against GM-CSF or its receptor develop PAP phenotype [4,5]. These autoantibodies had been considered as the causative agent for aPAP. Ten years later, Trapnell and his colleagues proved this hypothesis by reproducing PAP in nonhuman primates that were injected with purified GMAbs from patients with aPAP [6].

On the other hand, Svenson et al. first reported that GMAbs occurred consistently in pharmaceutically prepared intravenous immunoglobulin (IVIG) [7]. They also demonstrated that these autoantibodies showed high avidity with GM-CSF. As IVIG is produced through purification of IgG from pooled plasma obtained from normal subjects, the consistent presence of GMAbs suggested the occurrence in the sera of normal subjects. In this regard, Svenson et al. reported that GMAbs was rarely present in $0.3 \%$ plasma of normal subjects [7]. Alternatively, Bazin et al. demonstrated that artificial activation of cryptic IgG auto reactivity to GM-CSF for explanation that GMAbs are consistently present at low levels in IVIG [8].

On the other hand, a ubiquitous occurrence of autoantibodies to various cytokines in the sera of normal subjects has been reported [9]. We demonstrated that GMAbs occurred ubiquitously at low levels in the sera of healthy subjects [10]. Currently available sandwich ELISA method to quantify GMAbs is accurate and precise for the diagnosis of autoimmune PAP discriminating from the values in healthy individuals, of which the detailed method was described and the results were intensively evaluated [11]. However, these reports did not determine the accurate concentrations of GMAbs in the sera of healthy subjects because at very low levels, non-specific IgG binding to coated GMCSF cannot be ignored by the ELISA system. Thus, assay development to detect low levels of GMAbs in high specificity should be necessary to quantify the accurate concentration of GMAbs in the sera healthy individuals.

When we measure the concentrations of GMAbs in the sera of normal subjects accurately, we are also confronted with difficulties as GMAbs form immune complexes with intrinsic GM-CSF, and thus the complexes are not necessarily trapped by recombinant GM-CSF coated on the plate. To solve the problem, we developed a liquid phase highsensitivity assay using chemiluminescence reaction that was able to detect GMAbs even in the form of immune complex. Subsequently, we estimated the true concentration of GMAbs by subtracting non-specific GM-CSF binding IgG from total GM-CSF binding IgG.

The concentrations of GMAbs in the sera of normal subjects measured by this novel method were lower than those estimated by the conventional ELISA in the previous reports $[10,11]$.

\section{Materials and Methods}

\section{Subjects}

The institutional review board of the Niigata University Medical \& Dental Hospital approved this study. All participants gave written informed consent in accordance with the Declaration of Helsinki. Both thirty normal subjects and patients with autoimmune PAP patients were enrolled in the study. Healthy subject group included 21 male and 9 female with median age was 44.5 (22-64).

*Corresponding author: Koh Nakata, Bioscience Medical Research Center, Niigata University Medical and Dental Hospital, Asahimachi-dori, Niigata, Japan, Tel: +81-25-227-0847; Fax: +81-25-227-0377; E-mail: radical@med.niigata-u.ac.jp

Received October 30, 2017; Accepted November 14, 2017; Published November 21, 2017

Citation: Shiiya K, lizuka M, Ito Y, Utako S, Atsushi H, et al. (2017) Estimation of the True Granulocyte-Macrophage Colony-Stimulating Factor (GM-CSF) Autoantibody Concentration in the Sera of Normal Subjects. J Bioanal Biomed 9: 272-276. doi:10.4172/1948-593X.1000192

Copyright: @ 2017 Shiiya K, et al. This is an open-access article distributed under the terms of the Creative Commons Attribution License, which permits unrestricted use, distribution, and reproduction in any medium, provided the original author and source are credited. 
Citation: Shiiya K, lizuka M, Ito Y, Utako S, Atsushi H, et al. (2017) Estimation of the True Granulocyte-Macrophage Colony-Stimulating Factor (GMCSF) Autoantibody Concentration in the Sera of Normal Subjects. J Bioanal Biomed 9: 272-276. doi:10.4172/1948-593X.1000192

\section{Preparation of standard GMAbs}

GMAbs were purified from pooled sera from 30 patients with aPAP as previously described [3]. The purity of isolated GMAbs was estimated to be $82.8 \%$ using ELISA (Bethyl Laboratories Inc., TX). Serum IgG fractions were purified by protein A/G Sepharose (GE Healthcare, UK) according to the manufacturer's instructions.

\section{bGM-CSF}

The rhGM-CSF (salgramostim) was purchased from GenzymeSanofi Corporation (Cambridge, MA) and biotinylated using the NHSPEO-biotin kit and monomeric avidin kit (Pierce Biotech, Rockford, IL, USA) according to the manufacturer's instructions. The purity of bGM-CSF was almost $100 \%$.

\section{Artificially formed immune complex}

Purified GMAbs $(100 \mathrm{ng} / \mathrm{ml})$ were incubated with $2.5 \mathrm{ng} / \mathrm{ml}$ of rhGM-CSF overnight at $4^{\circ} \mathrm{C}$ to form immune complex.

\section{Measurement of GMAbs concentration: Conventional method}

Serum GMAbs levels were measured using direct ELISA as previously reported. In brief, micro-ELISA plates (Maxisorp ${ }^{\mathrm{Tm}}$ flatbottom, clear, 96-well plates; Nunc, Roskilde, Denmark) were coated with rhGM-CSF at $1 \mu \mathrm{g} / \mathrm{ml}$ with phosphate-buffered saline (PBS) at $4^{\circ} \mathrm{C}$ overnight. After washing with PBS containing $0.1 \%$ Tween ${ }^{n t}$ Twenty (0.1\% PBS-T) (MP Biomedicals, Solon, OH, USA), plates were stabilized with blocking solution (Stabilcoat ${ }^{\mathrm{Tw}}$, SurModics, Eden Prairie, MN, USA) for $1 \mathrm{~h}$ at room temperature. Human monoclonal GMAb that was kindly provided by Dr. Kenzo Takada (Evec Co. Ltd., Sapporo, Japan) was diluted in PBS containing $1 \%$ bovine serum albumin (1\% BSA/ PBS) and was used as a standard for antibody measurement. Serum samples were diluted into $1 \%$ BSA/PBS at a 100 -fold dilution, and a volume of $50 \mu \mathrm{l}$ of prepared samples and standard was transferred to plates. After keeping plates at room temperature for $1 \mathrm{~h}$ and washing with $0.1 \%$ PBS-T, GMAbs captured by rhGM-CSF were detected by peroxidase-labeled anti-human $\mathrm{Fc} \gamma$ (Dako Corporation, Carpinteria, CA, USA). After washing, color was developed using tetramethylbenzidine (TMB) solution, and the absorbance was measured at $450 \mathrm{~nm}$. The mean optical density and the standard deviation at $450 \mathrm{~nm}$ were shown in the Table 1 for various concentrations of standard human monoclonal antibody (Table 1).

\section{Measurement of GM-CSF binding IgG concentration by a novel method}

To detect low concentrations of GMAbs in the sera of normal

\begin{tabular}{|c|c|c|c|c|}
\hline Conc ng/mL & Mean OD & $\begin{array}{c}\text { Mean OD- } \\
\text { mean blank }\end{array}$ & SD & CV\% \\
\hline 1.5625 & 972 & 627 & 12 & 1.87 \\
\hline 0.78125 & 647 & 302 & 8 & 2.71 \\
\hline 0.390625 & 513 & 168 & 5 & 3.07 \\
\hline 0.1953125 & 428 & 83 & 8 & 9.03 \\
\hline 0.09765625 & 395 & 50 & 5 & 10.95 \\
\hline 0.048828125 & 367 & 22 & 8 & 37.68 \\
\hline 0.0244140625 & 357 & 12 & 8 & 69.99 \\
\hline 0 & 345 & 0 & 5 & - \\
\hline
\end{tabular}

Table 1: Concentration response of the calibration curve and accuracy of measuring the standard monoclonal GM-CSF autoantibody in the conventional ELISA system. Conc: Concentration of standard monoclonal GM-CSF autoantibody, J152-4C. OD: Optical Density, SD: Standard deviation, CV: Coefficient of Variation. subjects, we developed a novel method as follows (Figure 1). Briefly, each $50 \mu \mathrm{l}$ of sera from healthy subjects was diluted with $1 \% \mathrm{BSA} / \mathrm{PBS}-\mathrm{T}$ at 100-fold and incubated with excess amount of bGM-CSF (50 ng/ $\mathrm{ml}$ ) overnight at $4^{\circ} \mathrm{C}$ and transferred into 96 well streptavidin coated plate (Immobilizer streptavidin F96 White, Nunc, Roskilde, Denmark) previously hydrated with PBS. The plate trapped GMAbs bound with bGM-CSF for $1 \mathrm{~h}$ at room temperature. After washing the plate with PBS-T five times, each well was blocked with $100 \mu$ lof Stabilicoat for 1 $\mathrm{h}$, and further washed with PBS-T five times.

GMAbs captured by bGM-CSF were detected by alkaline phosphatase-labeled anti-human Fc $\gamma$ (Dako Corporation, Carpinteria, CA, USA). After washing, the activity of alkaline phosphatase was detected by CDP-Star (Applied Biosystems, CA) using a chemiluminescence plate reader (Berthold. Tec. GmbH \& Co. Germany). For standard GMAbs, purified polyclonal GMAbs described above was used for this assay.

\section{Statistical analysis}

Test for normality of continuous variables and homogeneity of variance were performed using Shapiro-Wilk test and Leuven test, respectively. Normally distributed continuous variables were reported as mean \pm standard error and data that were not normally distributed were reported as medians and interquartile ranges (median $25 \%, 75 \%)$. The paired $t$ test was used for comparisons between normally distributed data and the treatment periods. Comparisons of nonparametric data were made using the Wilcoxon signed-rank test. For group comparisons, unpaired t tests and Wilcoxon rank-sum tests were used. Correlation coefficient between two continuous variables was obtained using Spearman's correlation method. P values less than 0.05 were considered statistically significant.

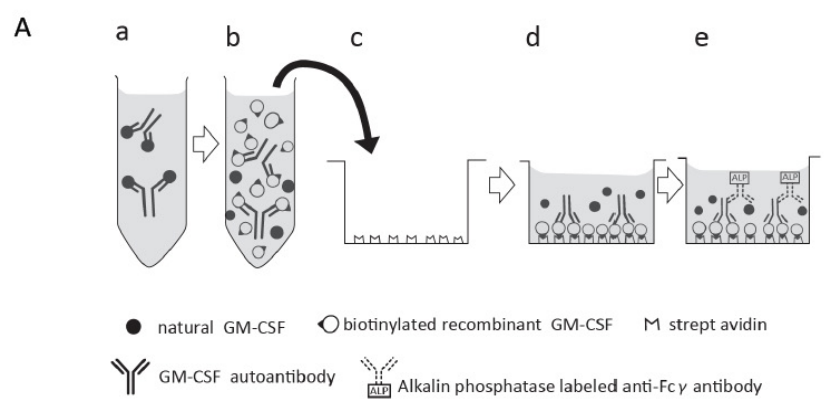

B

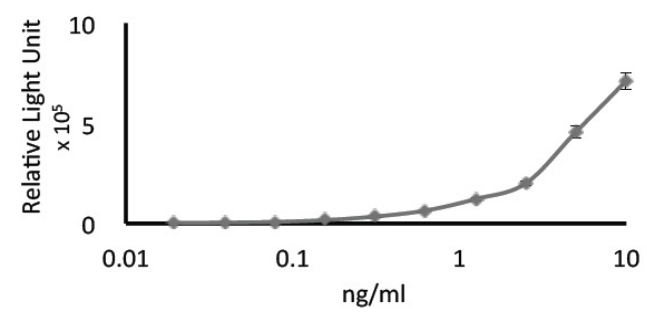

Figure 1: A: A schematic explanation for the novel ELISA system measuring GMAbs concentration in the sera from normal subjects. Diluted sera from healthy subjects was incubated with excess amount of bGM-CSF and transferred into 96 well streptavidin coated plate. The plate trapped GMAbs bound with bGM-CSF. GMAbs captured by bGM-CSF was detected by alkaline phosphatase-labeled anti-human Fcy. The activity of alkaline phosphatase was detected by chemiluminescence plate reader. B: A standard plot of the relative light unit provided by the chemiluminescence reaction at various concentrations of standard polyclonal antibody. 
Citation: Shiiya K, lizuka M, Ito Y, Utako S, Atsushi H, et al. (2017) Estimation of the True Granulocyte-Macrophage Colony-Stimulating Factor (GMCSF) Autoantibody Concentration in the Sera of Normal Subjects. J Bioanal Biomed 9: 272-276. doi:10.4172/1948-593X.1000192

\section{Results}

\section{A novel method for measuring GM-CSF binding IgG}

To estimate the low concentration of GMAbs in the healthy subjects which forms immune complex with intrinsic GM-CSF [10], we developed a novel method in which low concentration of GMAbs are trapped with excess concentration of bGM-CSF followed by capture on streptavidin-coated plate and then binding with alkaline phosphatase labeled anti-IgG Fc antibody followed by chemiluminescence reaction to detect alkaline phosphatase activity (Figure 1A). The standard curve of purified polyclonal GMAbs appeared a downward convex shape (Figure 1B). When we determine the detection limit as the end concentration with CV less than $10 \%$, the lower detection limit for polyclonal standard was $0.02 \mathrm{ng} / \mathrm{ml}$ (Table 2). In contrast, when the lower detection limit was determined using the conventional method as described in "Material and Methods", it was $0.195 \mathrm{ng} / \mathrm{ml}$, indicating the sensitivity of the novel method was 10 fold higher than that of the conventional method (Table 1).

\section{Feasibility of the novel method to measure the concentration of GMAbs in the immune complex}

Artificially formed immune complex described in the Materials and Methods (containing $2.5 \mathrm{ng} / \mathrm{ml}$ standard purified GMAbs) was incubated with various concentrations of bGM-CSF $(0-25 \mathrm{ng} / \mathrm{ml})$ and the concentration of bound GMAbs was measured by the novel method, The concentration was dose-dependently increased as the concentration of added bGM-CSF reaching $2.5 \mathrm{ng} / \mathrm{ml}$ of GMAbs at $12.5 \mathrm{ng} / \mathrm{ml}$ of bGM-CSF (Figure $2 \mathrm{~A}$ ). On the other hand, when variable concentrations of GMAbs in artificially formed immune complex were measured by the novel method, the values were almost compatible with the values for GMAbs alone $(0-5 \mathrm{ng} / \mathrm{ml})$ and slightly lower with $6 \%$ reduction at $10 \mathrm{ng} / \mathrm{ml}$ of GMAbs (Figure $2 \mathrm{~B}$ ). These results suggest that the novel method is able to estimate the concentration of GMAbs even in the form of immune complex.

\section{Estimation of the true GMAbs concentration}

To estimate the true concentration of GMAbs in the sera of normal subjects, we need to know the concentration of IgG that bind with GM-
CSF nonspecifically. When the 100 -fold diluted serum was incubated with various concentrations of non-labeled GM-CSF $(0-10 \mathrm{mg} / \mathrm{ml})$ and bGM-CSF $(50 \mathrm{ng} / \mathrm{ml})$ and then the concentration of $\operatorname{IgG}$ bound with bGM-CSF was measured by trapping with streptavidin coated plate, the total IgG bound bGM-CSF reduced as the added non-labeled GM-CSF increased, reaching a bottom that is presumed to be the concentration of IgG bound nonspecifically (Figure 3). The inhibition rate of the bound IgG by the added non-labeled GM-CSF appeared a saturationbinding curve (Figure $3 \mathrm{~A}$ ). The Michaelis-Menten plot provided a $\mathrm{V}_{\max }$ value that was considered to be the ratio of true GMAbs concentration per total IgG bound bGM-CSF (Figure 3B).

\section{Distribution of true GMAbs concentrations in 30 normal subjects}

Based on each $\mathrm{V}_{\text {max }}$ value and each total IgG concentration bound bGM-CSF (Figure $4 \mathrm{~A}$ ), each true GMAbs concentration was calculated. In 30 normal subjects, the value was estimated to be 10.6 to $799.5 \mathrm{ng} /$ $\mathrm{ml}$ with median value at $52.6 \mathrm{ng} / \mathrm{ml}$ (Figure $4 \mathrm{~B}$ ). As the lower detection

\begin{tabular}{|c|c|c|c|c|}
\hline Conc ng/ml & Mean RLU & $\begin{array}{c}\text { Mean RLU- } \\
\text { mean blank }\end{array}$ & SD & CV\% \\
\hline 10 & 711697 & 711033 & 42461 & 5.97 \\
\hline 5 & 457907 & 457243 & 30663 & 6.71 \\
\hline 2.5 & 201140 & 200477 & 11532 & 5.75 \\
\hline 1.25 & 122350 & 121687 & 9216 & 7.57 \\
\hline 0.625 & 64927 & 64263 & 1849 & 2.88 \\
\hline 0.3125 & 35243 & 34580 & 1449 & 4.19 \\
\hline 0.15625 & 17280 & 16617 & 1366 & 8.22 \\
\hline 0.078125 & 8440 & 7777 & 366 & 4.70 \\
\hline 0.0390625 & 5087 & 4423 & 125 & 2.83 \\
\hline 0.01953125 & 3000 & 2337 & 121 & 5.17 \\
\hline 0.009765625 & 1850 & 1187 & 161 & 13.54 \\
\hline 0 & 663 & 0 & 57 & - \\
\hline
\end{tabular}

Table 2: Concentration response of the calibration curve and accuracy of measuring the standard polyclonal GMAbs in the novel ELISA system. Conc: Concentration of standard polyclonal GMAbs that was prepared from pooled sera of patients with autoimmune PAP as described in Materials and Methods. RLU: Relative Light Unit, SD: Standard deviation, CV: Coefficient of variation.
A

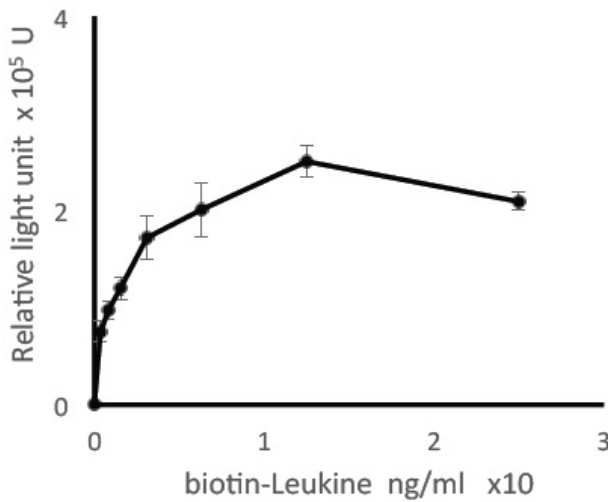

B

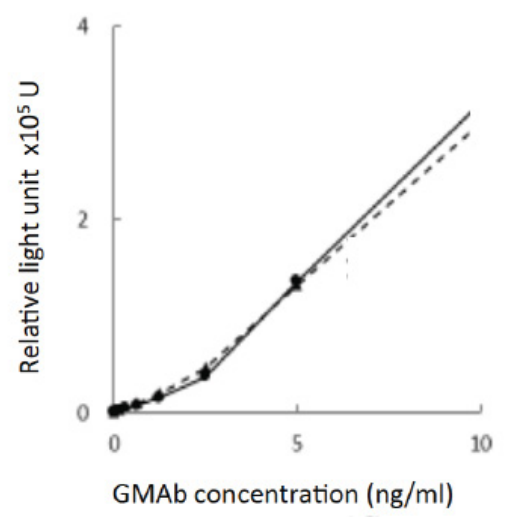

Figure 2: Binding of artificial GM-CSF/GMAbs complex with bGM-CSF. Binding activity is expressed as the relative light units in chemiluminescence reaction. A: Binding of artificial GM-CSF/GMAbs complex containing $2.5 \mathrm{ng} / \mathrm{ml}$ of the antibody described in Material and Methods with various concentrations of bGM-CSF (0-2.5 $\mathrm{ng} / \mathrm{ml}$ ). B: Binding of various concentrations (0-10 $\mathrm{ng} / \mathrm{ml}$ ) of GMAbs (solid line) or artificial GM-CSF/GMAbs complex (dotted line) with bGM-CSF (50 $\mathrm{ng} / \mathrm{ml})$. 
Citation: Shiiya K, lizuka M, Ito Y, Utako S, Atsushi H, et al. (2017) Estimation of the True Granulocyte-Macrophage Colony-Stimulating Factor (GMCSF) Autoantibody Concentration in the Sera of Normal Subjects. J Bioanal Biomed 9: 272-276. doi:10.4172/1948-593X.1000192

A

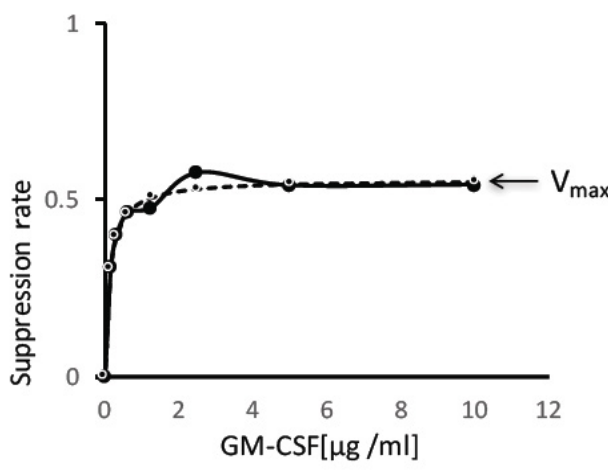

B

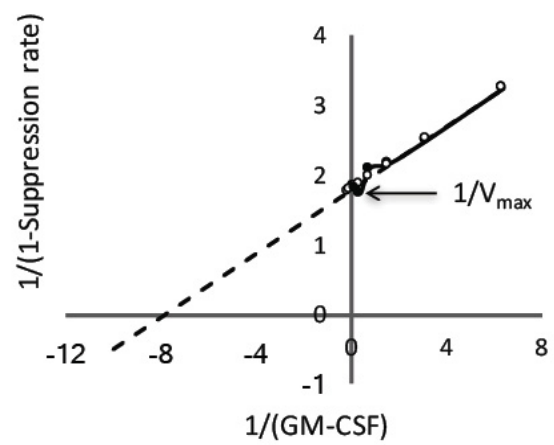

Figure 3: A: A typical curve for suppression rate of lgG binding with bGM-CSF in the 100 fold diluted serum of a normal subject by increasing concentration of non-labeled GM-CSF $(0-10 \mathrm{mg} / \mathrm{ml})$. The vertical axis indicates the inhibition rate of IgG binding activity per IgG binding activity without non-labeled GMCSF. B: The Michaelis-Menten plot of $A$. The Vmax value is considered to be the ratio of true GMAbs concentration per total IgG bound bGM-CSF.

limit for the polyclonal standard was $0.02 \mathrm{ng} / \mathrm{ml}$ and the serum samples were diluted at 100 -fold, the lower detection limit was $2.0 \mathrm{ng} / \mathrm{ml}$. Thus, all of 30 samples were positive for GMAbs with mean value of $164.0 \mathrm{ng} /$ $\mathrm{ml}$. These data suggested that the novel method revealed that the true GMAbs concentrations were lower than the value reported previously $((0.28[0.20-0.51] \mu \mathrm{g} / \mathrm{ml} ; \mathrm{n}=38)[11]$.

\section{Validation of the data obtained by the novel ELISA method}

To evaluate the accuracy of the novel ELISA system, we compared the GMAbs concentrations in the same 30 normal subjects between the novel and conventional ELISA system. Of all, 13 samples were under the detection limit in the conventional method $(19.5 \mathrm{ng} / \mathrm{ml}$, Table 1$)$, whereas all samples were over the detection limit in the novel method (2 $\mathrm{ng} / \mathrm{ml})$. Thus, only 17 data were available in the conventional method, there was still a strong correlation between the novel and conventional methods (Figure 5, $\rho=0.738, \mathrm{P}=0.001$ ), suggesting the reliability of the novel method.

\section{Discussion}

In this study, we developed a novel quantitation system for measuring the low concentration of GMAbs in the sera of normal subjects. The system demonstrated that low concentrations of GMAbs were detected in 30 of 30 normal subjects, whereas 17 of 30 subjects by the conventional ELISA system. The accuracy and reliability of the data obtained by the novel quantitation system was confirmed by comparing the data with those obtained by the conventional method. The presence of GMAbs in the blood of normal subjects was first demonstrated by Svenson et al. in 1998 [7], who reported that IgG type of GMAbs were consistently detected in every batch of pharmaceutically prepared

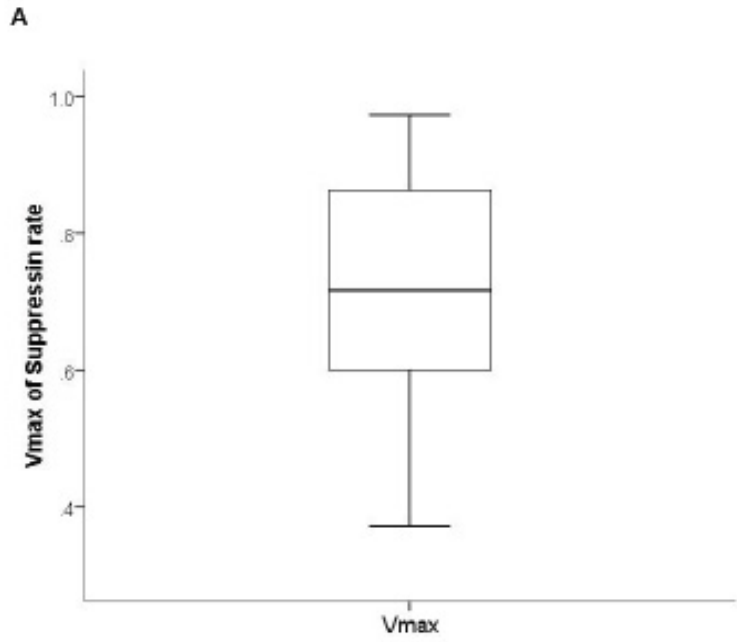

B

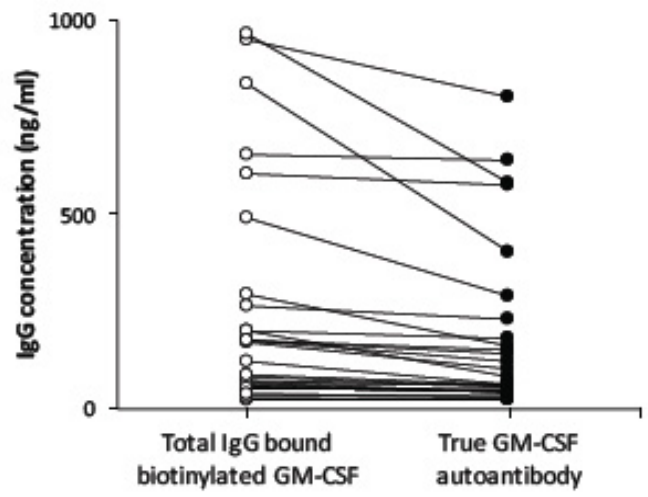

Figure 4: A: Distribution of $V \max$ values that means the ratio of true GMAbs concentration per total IgG bound bGM-CSF in the sera of 30 normal subjects. B: A parallel plot of concentrations for the total IgG bound with bGM-CSF and the true GMAbs.

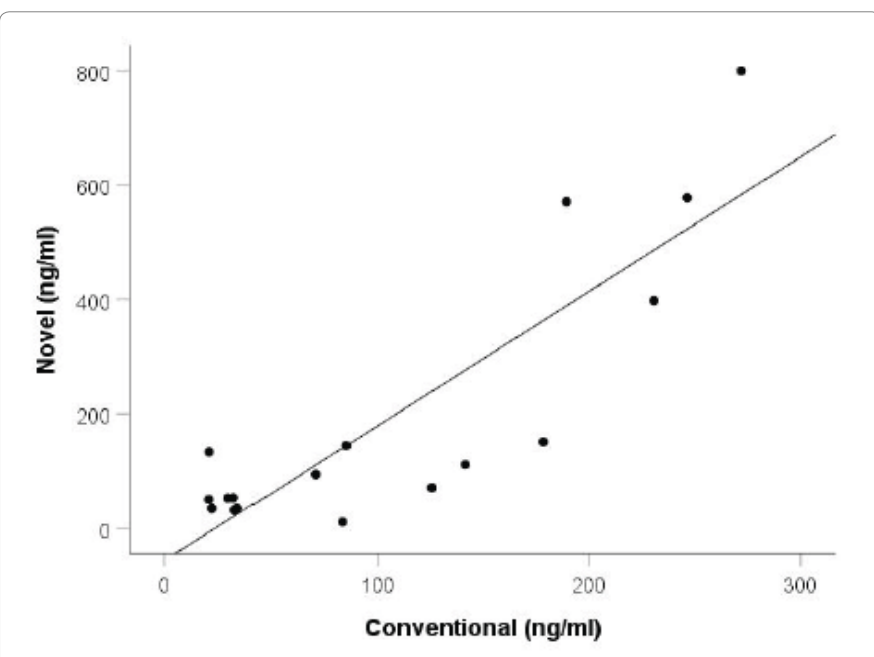

Figure 5: Correlation of GMAbs concentrations determined by the novel method with those determined by the conventional method. By Spearman's rank correlation method, $\rho$ value was $0.738(n=17$ and $P=0.001)$. 
Citation: Shiiya K, lizuka M, Ito Y, Utako S, Atsushi H, et al. (2017) Estimation of the True Granulocyte-Macrophage Colony-Stimulating Factor (GMCSF) Autoantibody Concentration in the Sera of Normal Subjects. J Bioanal Biomed 9: 272-276. doi:10.4172/1948-593X.1000192

immunoglobulin preparation. Although the occurrence of GMAbs in the normal sera was thought to be infrequent, but IVIG is produced from pooled plasma of several thousands of healthy donors, and thus, Svenson et al. demonstrated that the consistent detection of GMAbs in every IVIG might reflect the presence of relatively high concentration of GMAbs in a few donors [7]. On the other hand, we previously thought that low concentration of GMAbs was consistently present in the sera of all normal subjects using conventional ELISA method and immunoblotting for some part of subjects. In that study, we did not mention about nonspecific binding of IgG other than GMAbs with GMCSF coated on the ELISA plate. In this regard, Meager et al. submitted a criticism to our report that nonspecific binding of IgG other than GMAbs might affect the results of measuring GMAbs concentrations in the sera of normal subjects [12]. According to their criticism, we evaluated the nonspecific binding of IgG other than GMAbs. We realized that the nonspecific binding was not negligible in normal subjects in whom the true concentration of GMAbs was expected to be very low, and thus, we applied a high sensitivity detection system to measure even low concentrations of GMAbs in nano gram order.

Because pretreatment of the serum is likely to cause artificial activation of cryptic IgG that may bind GM-CSF [8], we did not perform any pretreatment on the sera with acid or detergent to dissociate GMCSF/GMAbs immune complex. Our data showed that liquid-phase preincubation with excess amount of extrinsic bGM-CSF efficiently substituted the intrinsic GM-CSF in the complex.

In the previous report, bound GM-CSF in the immune complex of normal sera was estimated to be approximately $3 \mathrm{ng} / \mathrm{ml}$ in average [10], whereas the mean concentration of GMAbs was $164.0 \mathrm{ng} / \mathrm{ml}$ in the present study. When the molecular weight of GM-CSF and IgG were presumed to be 23 and $150 \mathrm{kDa}$, respectively, the molar ratio of these molecules involved in the sera of normal subjects was approximately 1: 8.4. Anyway, the immune complex in the sera of normal subjects is consisted of little GM-CSF bound with excess GMAbs. The binding sites of GMAbs in the complex are likely not occupied completely, and thus, the free sites may easily bind to the extrinsic GM-CSF.

In the conventional method, the detection of GMAbs bound with GM-CSF coated on the plate was performed using peroxidase labeled anti-Fc gamma second antibody followed by color reaction with TMB solution. By this system, we can detect GMAbs concentration higher than $19.5 \mathrm{ng} / \mathrm{ml}$ as shown in this study. To increase the sensitivity of detection, we applied alkaline phosphatase labeled anti-Fc gamma second antibody followed by chemiluminescence reaction. This system detected as low as $2.0 \mathrm{ng} / \mathrm{ml}$ of GMAbs. Moreover, improvement in the detection sensitivity did not increase the detection rate of GMAbs in the sera of normal subjects, because the novel system estimates the binding of nonspecific IgG with GM-CSF in each serum independently.

GMAbs in healthy subjects were found to reduce GM-CSF stimulation of myeloid cells as reported previously [10]. Serum levels of GM-CSF and GMAbs may be exquisitely balanced in healthy individuals [10]. Namely, most serum GM-CSF is present in the form of an immune complex, and at levels much higher than the free GMCSF levels previously reported [10]. Thus, GMAbs in healthy subjects may play an indispensable physiological role to keep the homeostasis of cytokine environment. It may attenuate the bioactivity of excess GMCSF and eradicate the inflammation.

In conclusion, we confirmed the occurrence of serum GMAbs in healthy subjects, however, the concentration were lower than demonstrated previously. The overestimation in the previous study is likely due to the underestimation of nonspecific IgG binding in the healthy sera. We believe that the present study will lead physicians and researchers to proper understanding on the significance of GMAbs in healthy subjects.

\section{Acknowledgements}

We thank Kanji Uchida and Masato Watanabe for valuable suggestions and discussions

\section{Funding}

The Japan Society for the Promotion of Science supported this work in part with a grant from Category $15 \mathrm{H} 04829$ (K.N.), 15K15321(K.N.). Japan Agency for Medical Research and Development also provided a grant, 15ek0109079h0001 (KN) and 15Aek0109063h0002 (YI) to support this research.

\section{Conflict of Interest}

None of the authors has a financial relationship with a commercial entity with an interest in the subject of this manuscript.

\section{Reference}

1. Tanaka N, Watanabe J, Kitamura T, Yamada Y, Kanegasaki S, et al. (1999) Lungs of patients with idiopathic pulmonary alveolar proteinosis express a factor which neutralizes granulocyte-macrophage colony stimulating factor. FEBS Lett 442: 246-250.

2. Kitamura T, Tanaka N, Watanabe J, Uchida K, Kanegasaki S, et al. (1999) Idiopathic pulmonary alveolar proteinosis as an autoimmune disease with neutralizing antibody against granulocyte/macrophage colony stimulating factor. J Exp Med 190: 875-880.

3. Uchida K, Nakata K, Trapnell BC, Terakawa T, Hashimoto E, et al. (2004) Highaffinity autoantibodies specifically eliminate granulocyte-macrophage colonystimulating factor activity in the lungs of patients with idiopathic pulmonary alveolar proteinosis. Blood 103: 1089-1098.

4. Dranoff G, Crawford AD, Sadelain M, Ream B, Rashid A et al. (1994) Involvement of granulocytemacrophage colony-stimulating factor in pulmonary homeostasis. Science 264: 713-716.

5. Robb L, Drinkwater CC, Metcalf D, Li R, Ko"ntgen F, et al. (1995) Hematopoietic and lung abnormalities in mice with a null mutation of the common beta subunit of the receptors for granulocyte-macrophage colony-stimulating factor and interleukins 3 and 5. Proc Natl Acad Sci U S A 92: 9565-9569.

6. Sakagami T, Beck D, Uchida K, Suzuki T, Carey BC, et al. (2010) Patientderived granulocyte/ macrophage colony-stimulating factor autoantibodies reproduce pulmonary alveolar proteinosis in nonhuman primates. Am J Respir Crit Care Med 182: 49-61.

7. Svenson M, Hansen MB, Ross C, Diamant M, Rieneck K, et al. (1998) Antibody to granulocyte-macrophage colony-stimulating factor is a dominant anticytokine activity in human IgG preparations. Blood 91: 2054-2061.

8. Bazin R, St-Amour I, Laroche A, Lemieux R (2010) Activated cryptic granulocyte-macrophage colony-stimulating factor autoantibodies in intravenous immunoglobulin preparations. Blood 115: 431.

9. Watanabe M, Uchida K, Nakagaki K, Kanazawa H, Trapnell BC, et al. (2007) Anti-cytokine autoantibodies are ubiquitous in healthy individuals. FEBS Lett 581: 2017-2021.

10. Uchida K, Nakakta K, Suzuki T, Luisetti M, Watanabe M, et al. (2009) Granulocyte/macrophage colony-stimulating factor autoantibodies and myeloid cell immune functions in healthy subjects. Blood 113: 2547-2556.

11. Uchida K, Nakata K, Carey BC, Chalk T, Suzuki T, et al. (2014) Standardized serum GM-CSF autoantibody testing for the routine clinical diagnosis of autoimmune pulmonary alveolar proteinosis. J Immunol Methods 402: 57-70.

12. Meager A, Cludts I, Thorpe R, Wadhwa M (2010) Are neutralizing anti-GM-CSF autoantibodies present in all healthy persons? Blood 115: 433-434. 